\title{
Fluidic Injection Scenarios for Shock Pattern Manipulation in Exhausts
}

\author{
Bernhard Semlitsch* \\ Whittle Laboratory, Department of Engineering, University of Cambridge \\ 1 JJ Thomson Avenue, Cambridge, CBз ODY, United Kingdom \\ Mihai Mihăescu ${ }^{\dagger}$ \\ Linné FLOW Centre, Department of Mechanics, Royal Institute of Technology (KTH),
}

Osquars Backe 18, 10044 Stockholm, Sweden

\begin{abstract}
Screening numerically internal fluidic injection scenarios for the manipulation of the double diamond shock pattern in convergent-divergent nozzle exhausts, we demonstrate the individual importance of design parameters. We find that the evolving shock pattern is sensitive to the injection location, while the persistence of the induced counterrotating vortex pairs is primarily governed by the injection pressure. Injection close to the nozzle exit generates secondary vortical structures amplifying the fluctuations in the nozzle vicinity.
\end{abstract}

\section{Introduction}

The structure of shock pattern appearing in supersonic exhausts is determined by the nozzle contours and its operating conditions. For supersonic aircrafts, the nozzle adapts to the turbine operating condition optimising the thrust performance. The nozzle transformability challenges its design to reduce shock associated losses and noise. Chevrons have been employed at the nozzle trailing edge to diminish acoustic noise by inducing streamwise vortical structures. ${ }^{1}$ This shifts the intensest momentum mixing to initial shear layer development, while decreasing the extent of turbulent coherent structures towards the potential core region. However, chevrons block the flow reducing the thrust. 2 Fluidic injection can be used for the same purpose, allowing more flexible application with possibly less thrust penalty. The optimisation of the injection strategy spans over a variety of parameters, such as injector location $x_{i}$, number, shape, angle $\theta$, cross-section, and flow momentum. These design parameters may influence each other or be optimal at different nozzle operating conditions. Further, effort is required to drive fluidic injection. Therefore, we investigate different setups for fluidic injection numerically, reporting the impact on thrust performance, shock

${ }^{*} \mathrm{PhD}$, Research Associate.

${ }^{\dagger} \mathrm{PhD}$, Associate Professor. 
pattern strength, and the minimisation of the turbulence kinetic energy levels in the exhaust shear layer. Thereby, we provide the insight for fluidic injection design to achieve the desired outcome.

\section{Methodology}

The investigated biconical nozzle geometry is shown with the injection design parameters in Fig. 1. The nozzle is characterised by an area ratio of 1.23 (relating the nozzle exit to the nozzle throat) and a design Mach number of 1.56. The nozzle exit diameter, $D_{e}$, is $57.5 \mathrm{~mm}$ and the nozzle lip thickness is $0.5 \mathrm{~mm}$. Four injection locations, $x_{i}$, i.e. -0.1 $D_{e},-0.388 D_{e},-0.857 D_{e}$, and $-1.1 D_{e}$, upstream of the nozzle exit are considered, where the nozzle throat is located at $-1.03 D_{e}$. The injectors, with a diameter $D_{j}$ of $2.57 \mathrm{~mm}$, are distributed equidistantly around the circumference. Configurations with six, twelve and twenty-four injectors have been simulated. The inclination angles, $\theta$, of $30^{\circ}, 60^{\circ}, 90^{\circ}$, and $135^{\circ}$ relative to the nozzle centreline are considered. Additionally, thin slot configurations at the trailing edge of the nozzle are examined, where the cross-sectional area is equal to the cylindrical injectors. To fit the slot channels under the outer nozzle shell, the inclination angles restricted to $11^{\circ}$ and $22^{\circ}$.

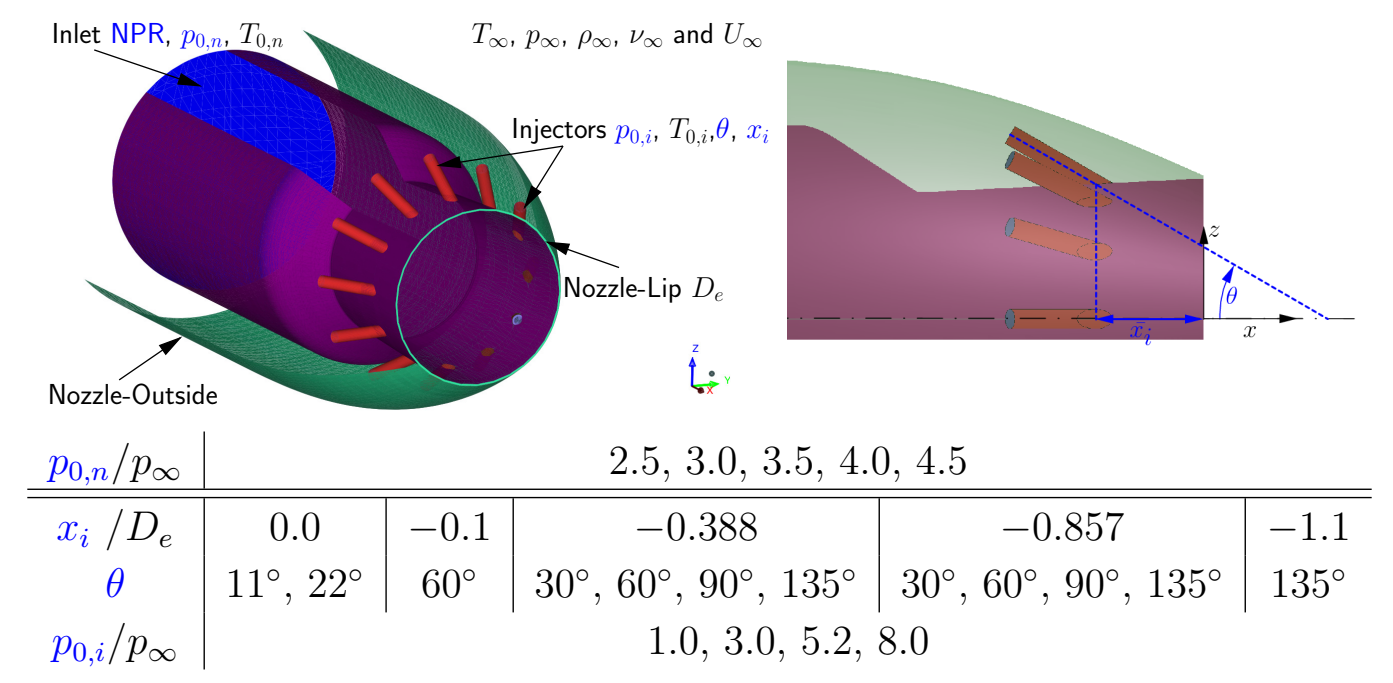

Figure 1. Nozzle geometry with injectors. The investigation parameters are indicated in blue and the configurations are tabulated.

The operating fluid is air being considered as ideal gas with an isentropic exponent of 1.4. The temperature relation of the dynamic viscosity is handled using Sutherland's formula. At the nozzle inlet, the total temperature is $367 K$ and the total pressure is set as ratio to the ambient pressure (i.e. Nozzle Pressure Ratio (NPR)) varied in the range of 2.5 to 4.5 . The injectors are supplied (at the ambient temperature) by a total pressure source referenced to the ambient pressure as the Injection Pressure Ratio (IPR) examined in the range of 3.0 to 8.0. The exhaust expands into ambient conditions moving with a free-stream Mach number of 0.1 . The ambient temperature is $288.16 \mathrm{~K}$ and the ambient pressure, $p_{\infty}$, is 1 atm.

The time-averaged, compressible Reynolds-Averaged Navier-Stokes equations are solved by a node-based, finite-volume solver. A third-order MUSCL scheme discretises the energy, 
density, and momentum terms. For the static pressure and the turbulence quantity terms, a second-order scheme is employed. The $k$ - $\omega$ SST turbulence closure has been selected for turbulence modelling. The simulations were considered as converged, when the normalised residuals decreased at least three orders of magnitudes. ${ }^{\text {a }}$ Block structured meshes have been used with a general O-grid structure and a total cell number of approximately seven million cells. Boundary layer refinement towards all walls is considered, where each injector is provided with an individual O-grid structure. The numerical domain extends over $8 D_{e}$ radially and $35 D_{e}$ downstream.

PIV measurements by Cuppoletti et. a ${ }^{3}$ are available without injection, which are compared to the numerical data in Fig. 2 for $p_{0, n} / p_{\infty}=4.0$. The shock pattern structure and the location of the outer Mach disk are captured well. The experiments reveal higher turbulence kinetic energy magnitudes downstream of the potential core and in a relatively confined shear layer. Further, the free stream turbulence levels in the experiments are higher than for the computational simulations. Figure 3 shows that also the co-flow velocity is slightly higher in the experimental setup. This dissimilarity contributes to the differences observed in the jet spread rate and the turbulence kinetic energy levels. Further, the high flow velocities result inherently in large forces and vibrations in the nozzle while running the experiment. Consequently, the vibrations provoke fluctuations in the boundary layer and affect the flow development. Nonetheless, the comparison shows good agreement in the shock pattern, while the shear layer spreads is over-predicted by the simulation indicating an over-estimated turbulent viscosity.
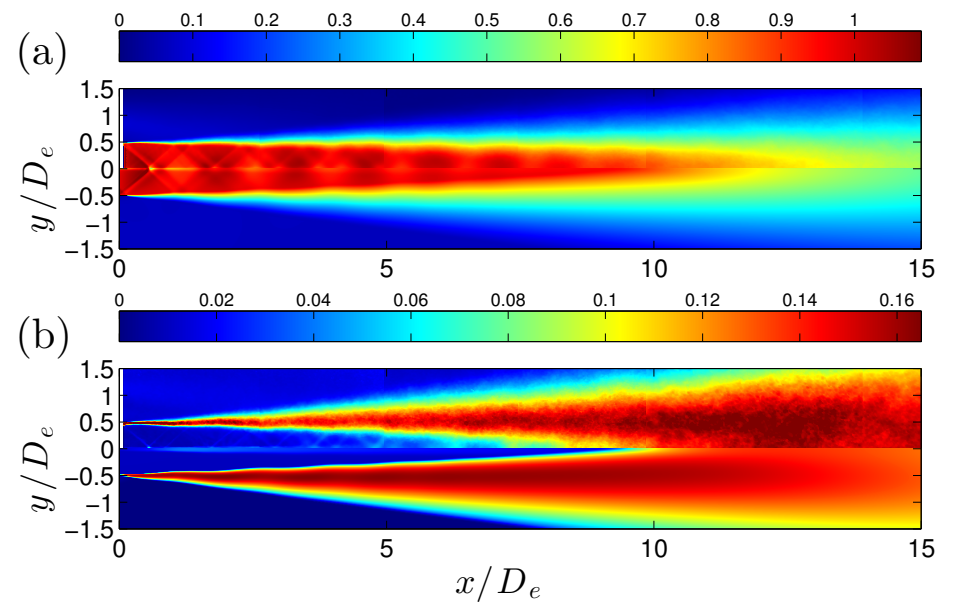

Figure 2. Comparison of the numerical results with PIV measurements, ${ }^{3}$ where the experimental flow visualisation is shown on the top and the numerical data on the bottom. The streamwise velocity normalised by the nozzle mean exit velocity, $\overline{u_{e, x}}$, is shown in (a) and the normalised turbulence kinetic energy, $\sqrt{T K E} / \overline{u_{e, x}}$, in (b). 


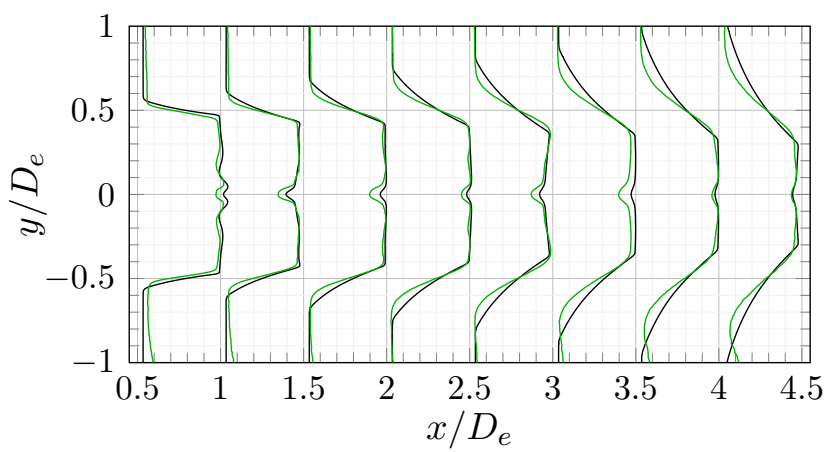

Figure 3. Normalised streamwise velocity profiles, $u_{x} / \overline{u_{e, x}}$, obtained by PIV measurements (green) and RANS simulations (black).

\section{Results}

The injection location governs its capability to modify the shock pattern and turbulence kinetic energy distribution as illustrated in Fig. 4. Without injection (Fig. 4 (a)), a double shock diamond pattern can be observed in the vicinity of the nozzle, which consists of a shock pattern arising in the nozzle and another manifesting at the nozzle trailing edge. Further downstream, the flow unsteadiness smears out the time-averaged density gradient.

Injection at the nozzle trailing edge via thin slots does not penetrate the exhaust (as shown Fig. 4 (b-c)), but dominates the shear layer in the nozzle vicinity. The injected jets confine the exhaust and cause thereby an intensified shock reflection of the inner nozzle shock pattern. Noteworthy is that the injection induced vortical structures form counterrotating pairs with the ones from the adjacent injector. Similarly, injection in the convergent nozzle section has little impact on the shock pattern, but influences the flow mixing in the exhaust shear layer, as shown quantitatively in Fig. 5 (b).

Figures 4 (d-g) show that the injection penetrates the core flow when applied upstream of the trailing edge. Bow shocks establish upstream of the injection (when installed in the divergent nozzle section) and displace thereby the shock pattern. Injection close or near to the nozzle trailing edge moves the shocks from the nozzle exit. Contrary to the other injection locations, the internal shock pattern can be reshaped when injecting (even with low $\left.p_{0, i} / p_{\infty}\right)$ at the nozzle throat.

Counterrotating vortex pairs evolve for each cylindrical injector. In the wake of the injection, secondary counterrotating vortex pairs form in the proximity of the nozzle surface. Right downstream of the nozzle trailing edge, these secondary vortical structures bulge outwards in the exhaust shear layer due to the counterrotation and the non-uniform pressure distribution. Figure 4 shows that the strength of the individual counterrotating vortex pairs is dependent on the injection location, where injection close to the nozzle training edge and close to the throat promote the strength of secondary or primary vortical structures, respectively.

Figure 5 (b) shows that the introduction of streamwise vortical structures reduces the turbulence kinetic energy levels towards the end of the potential core. Although, fluidic

\footnotetext{
${ }^{a}$ When convergence was not achieved, the unsteady, compressible Reynolds-Averaged Navier-Stokes equations have been solved instead with a constant time step of $10^{-6} s$ until the time-average was resolved.
} 
injection close to the nozzle exit exhibits the highest potential to decrease flow fluctuations, the turbulence kinetic energy levels are intense in the nozzle vicinity for such configurations due to the secondary vortical structures.

By translating the shock pattern with fluidic injection, the pressure oscillations in the exhaust can be amplified or smoothed as demonstrated in Fig. 5 (a). The lowest shock strength and hence, most level pressure distribution in the exhaust can be achieved by injection close to the nozzle throat.

For the manipulation of a shock pattern, the injected flow momentum perpendicular to the crossflow is essential. Thus, steepening the injection can compensate for lower injection

(a) no injection

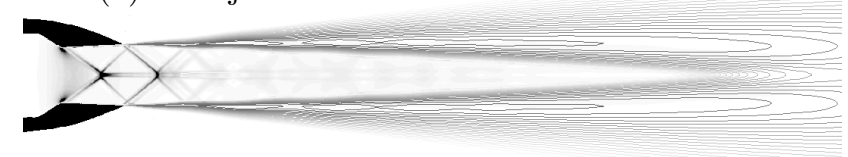

(b) $11^{\circ}$ lip injection
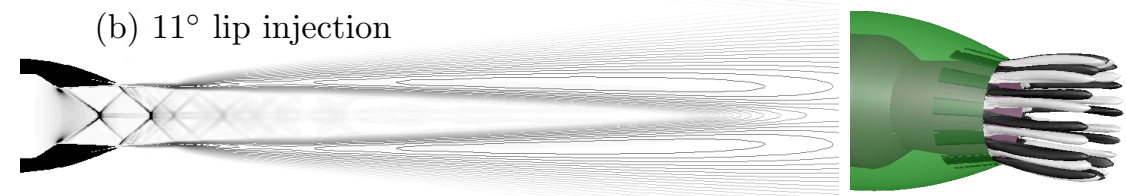

(c) 24 injectors at the lip with $22^{\circ}$
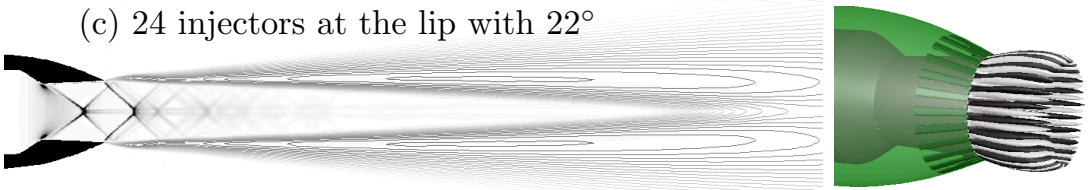

(d) $60^{\circ}$ trailing edge injection
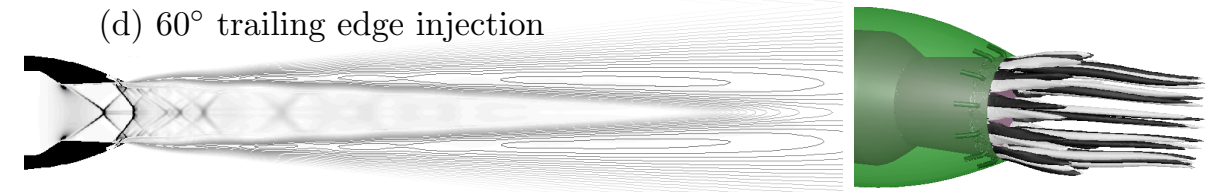

(e) $60^{\circ}$ near exit injection
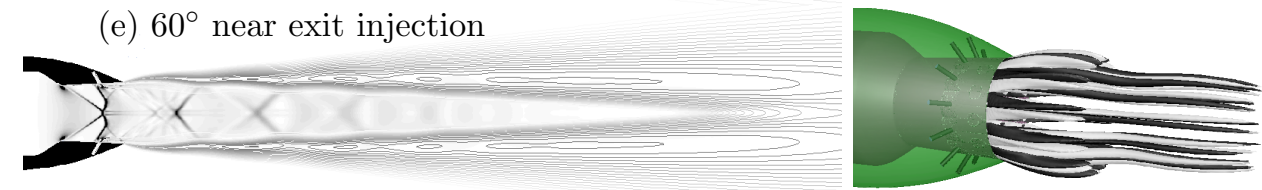

(f) $60^{\circ}$ near throat injection
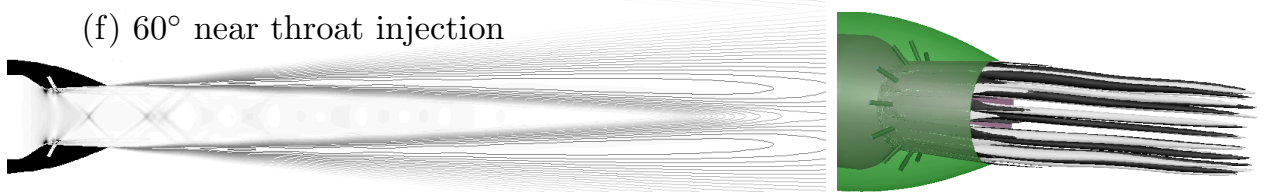

(g) $135^{\circ}$ up-throat injection
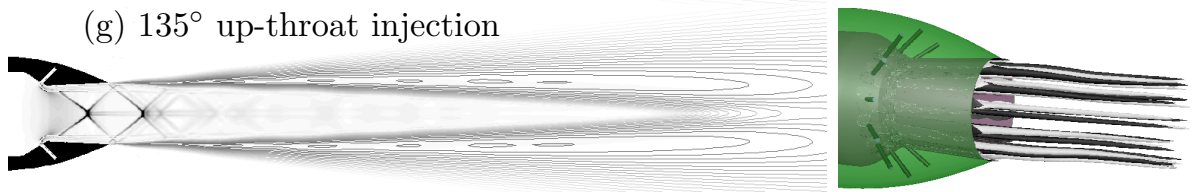

turbulence kinetic energy $\left(\mathrm{m}^{2} / \mathrm{s}^{2}\right)$

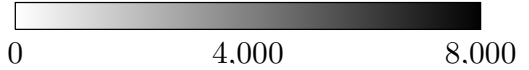

Figure 4. To the left, numerical Schlieren with the turbulence kinetic energy contour levels $\left(m^{2} / s^{2}\right)$ plotted on top; $\left(p_{0, n} / p_{\infty}=4.0\right.$ and $\left.p_{0, i} / p_{\infty}=5.2\right)$. To the right, iso-surfaces of the streamwise vorticity illustrate the vortex pairs (positive: white and negative: black). 

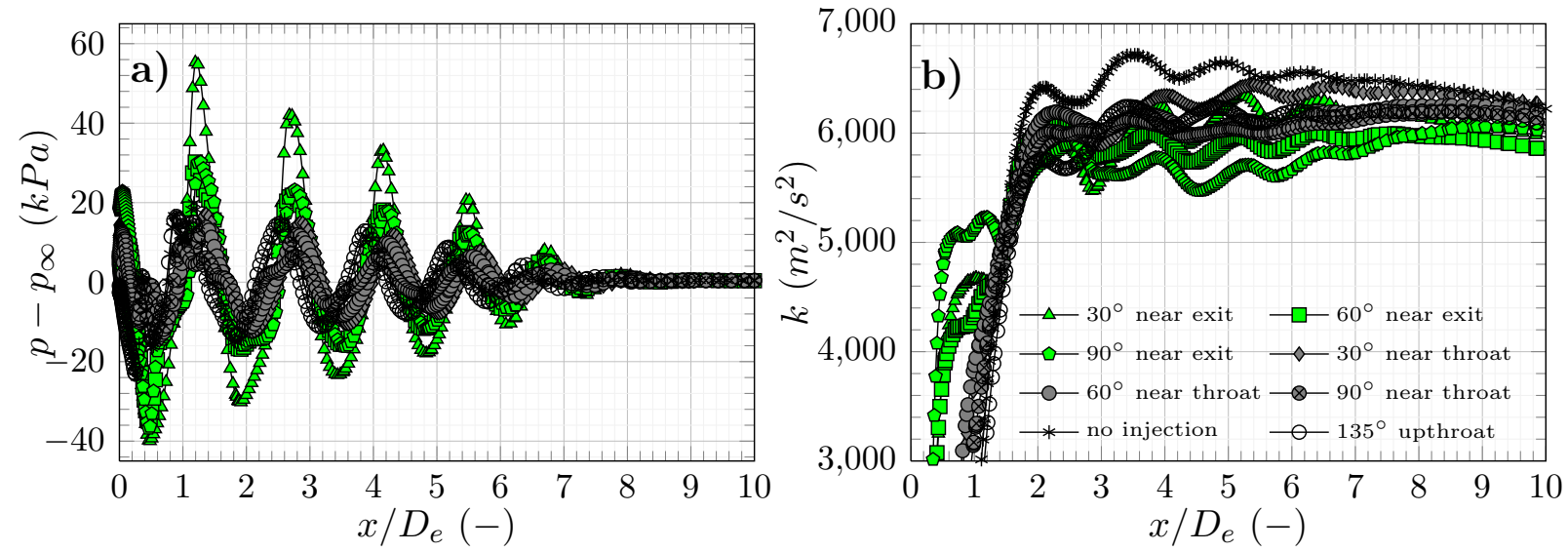

Figure 5. For $p_{0, n} / p_{\infty}=4.0$ and $p_{0, i} / p_{\infty}=5.2$, the static pressure, $p$, (a) and the turbulent kinetic energy, $k,(\mathbf{b})$ are plotted along axial lines at constant radius of $y=1 / 2 D_{e}$ and $y=1.1 D_{e}$, respectively.

pressure to achieve an equivalent shock pattern. Figure 6 exemplifies for configurations near the throat $(c-d)$ and the exit (a-b) that nearly identical shock pattern evolve when the

(a) near exit injection $30^{\circ}, p_{0, i} / p_{\infty}=5.2$
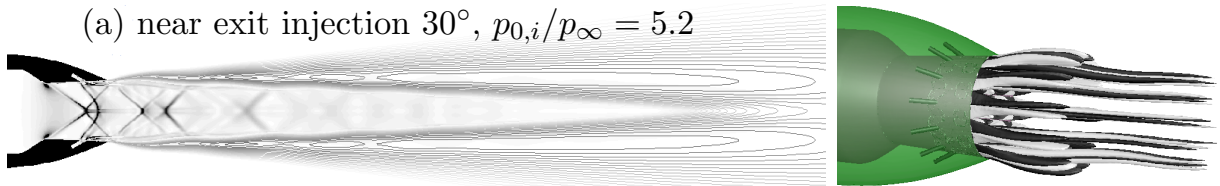

(b) near exit injection $60^{\circ}, p_{0, i} / p_{\infty}=3.0$
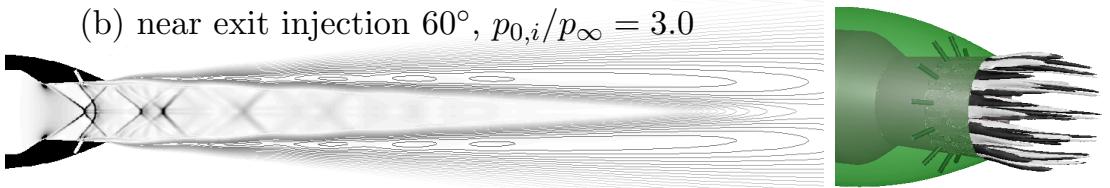

(c) $30^{\circ}$ near throat injection, $p_{0, i} / p_{\infty}=5.2$
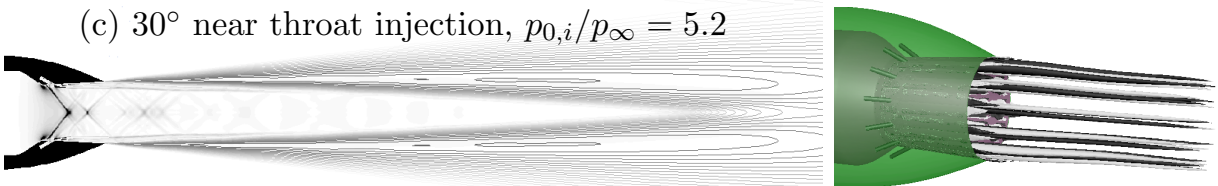

(d) $60^{\circ}$ near throat injection, $p_{0, i} / p_{\infty}=3.0$
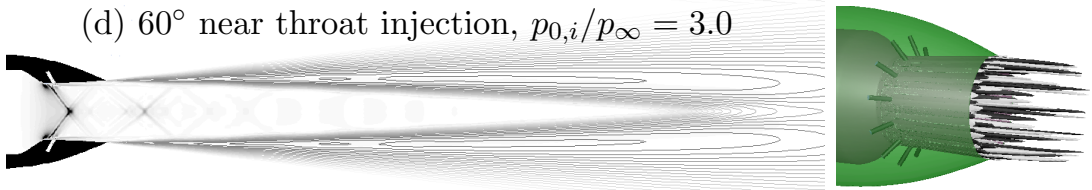

(e) $6 \times 60^{\circ}$ near throat injection, $p_{0, i} / p_{\infty}=5.2$
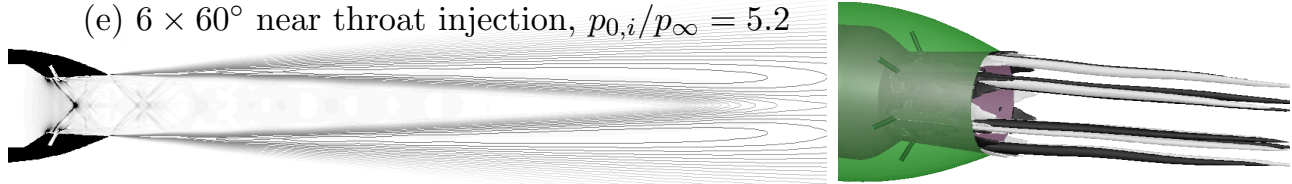

turbulence kinetic energy $\left(\mathrm{m}^{2} / \mathrm{s}^{2}\right)$

$0 \quad 8.000 \quad 800$

Figure 6. Same as Fig. 4; selected cases reveal trends with injection at $\left(p_{0, n} / p_{\infty}=4.0\right)$. 
injection pressure is reduced but the angle is increased.

The injection pressure primarily determines the persistence length of the streamwise vortical structures as shown in Fig. 6. Comparison with Fig. 4 clarifies the secondary role of the injection angle, where a steeper injection leads to longer streamwise vortical structures.

The vortical structures generated with six injectors prevail further downstream than with twelve injectors at the same configuration, which were shown in Fig. 6. (e) and Fig. 4 (f), respectively. This suggest that tight injector distribution influences the strength of adjacent counterrotating vortex pairs.

\section{Matching the Nozzle Exit Pressure}

The time-averaged static pressure profiles, shown in Fig. 7, reveal that fluidic injection can promote the pressure match at the nozzle exit, i.e. $p_{e}\left(y=D_{e} / 2\right)=p_{\infty}$. Especially injection close to the nozzle throat is beneficial at a nozzle pressure ratio of four to compensate for the over-expansion. At lower nozzle pressure ratios, the injection location near the nozzle exit has higher potential to rise the static pressure. However, the pressure distribution is irregularly perturbed by the injection closely placed to the nozzle exit. Comparing the profiles for injection at $p_{0, i} / p_{\infty}$ of 3.0 and 4.0 illustrates that the static pressure distribution changes drastically. Thus, the injected flow itself changes the static pressure distribution insubstantially, whereas the translation of the shock pattern dominates.

\section{Thrust performance}

Figure 8 reveals the impact of fluidic injection on the efficiency to generate specific thrust, $\mathcal{T}$. The effort of injection is incorporated in the thrust estimation as bleeding off the compressor,
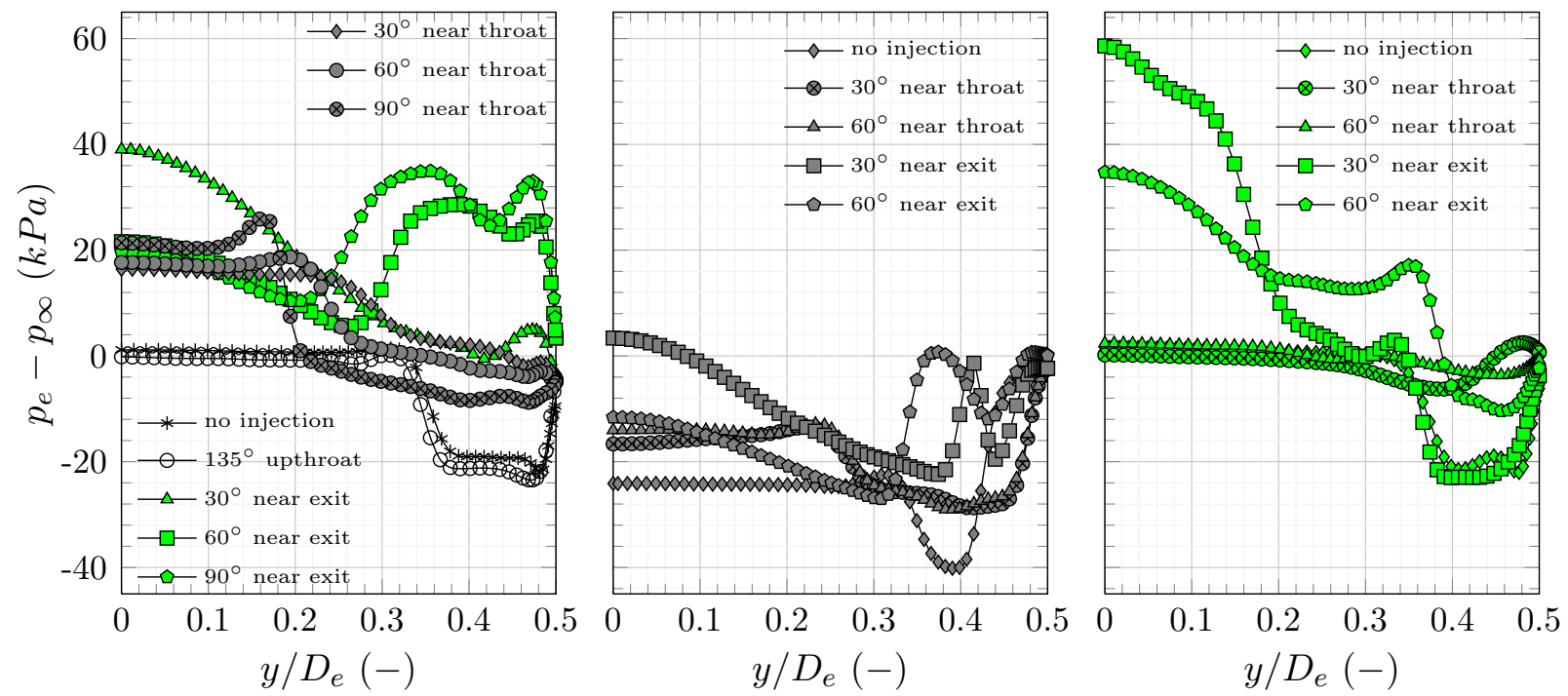

Figure 7. Effect of injection parameters on the static pressure distribution at the nozzle exit, $p_{e}$, shown for $p_{0, n} / p_{\infty}=4.0$ and $p_{0, i} / p_{\infty}=5.2$ to the left, for $p_{0, n} / p_{\infty}=3.0$ and $p_{0, i} / p_{\infty}=5.2$ in the mid, and $p_{0, n} / p_{\infty}=4.0$ and $p_{0, i} / p_{\infty}=3.0$ to the right. 
thus accounting for the injected momentum thrust,

$$
\mathcal{T}=\frac{\int_{A_{e}}\left(\rho_{e} v_{e, x}^{2}+p_{e}-p_{\infty}\right) d A}{\int_{A_{n}} \rho_{n} v_{n, x}^{2} d A+\int_{A_{j}} \rho_{j} v_{j, \theta}^{2} d A},
$$

where $p$ is the pressure, $v$ the velocity, $A$ the cross-sectional area, and $\dot{m}=\rho v A$ is the mass-flow with $\rho$ being the density. The indices, $e, n, j$ reference the variables to the nozzle exit, nozzle inlet, and injector inlet, respectively. For constant injection pressures, the injected mass-flow varies remarkably with respect to the nozzle operating conditions, while differing less in between injection approaches. With high injection pressures, the injected flow momentum is high (e.g. 10 to $20 \%$ of the nozzle flow momentum for $p_{0, i} / p_{\infty}=5.2$ ) and hence, influences significantly the thrust performance. Thus, shallow injection angles, conserving the momentum, perform generally better. Only for far over-expanded operating conditions, fluidic injection with high injection pressures becomes efficient. For optimisation of the thrust performance, it is beneficial to shift high velocities towards the nozzle exit, since the momentum dominates over the pressure thrust contribution. For acoustic considerations, the shock strength should be minimised to reduce its associated noise components and hence, $p_{e}\left(y=D_{e} / 2\right)=p_{\infty}$ should be fulfilled. Noteworthy is the potential of the $60^{\circ}$ injection close to the nozzle throat. The shock associated losses are reduced for this injection operating condition such that the steep injection angle losses diminish compared to the shallower equivalents.
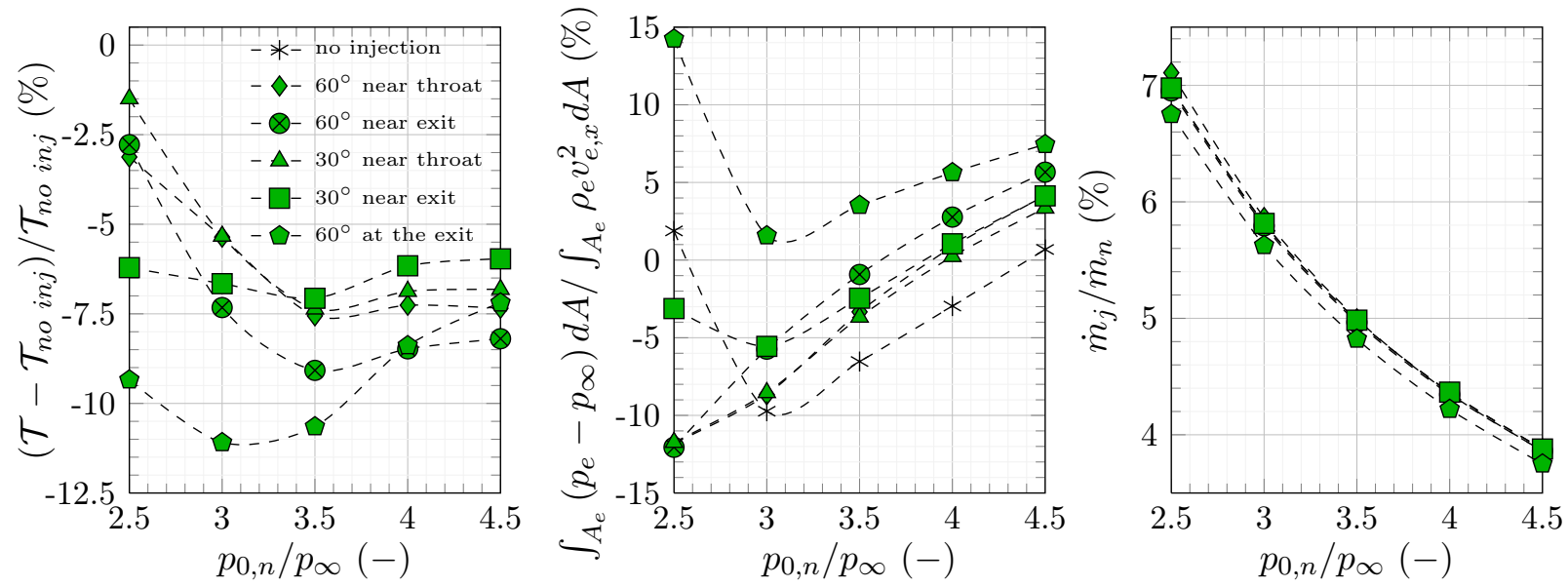

Figure 8. Thrust benefit (compared to the case without injection), pressure thrust, and massflow rate are shown as function of the nozzle operating condition with an injection pressure ratio of 5.2. (The counterintuitive trend of the pressure thrust at $p_{0, n} / p_{\infty}=2.5$ manifests due to the exhaust separating before the nozzle exit.)

\section{Conclusions}

Fluidic injection strategies into biconical nozzles are explored numerically. The computational method has been validated against experimental measurements showing that the shock pattern can be reliably captured, while the turbulent viscosity is over-predicted. Concluding we find that the following aspects should be considered designing fluidic injection;

8 of 9 
- Injection is most effectively employed close to the shock origin. Thus, the injection location was found most important optimisation parameter to manipulate the shock pattern. Only injection close to the throat allows manipulation of the nozzle internal shocks.

- The flow momentum perpendicular to the crossflow is the important parameter to manipulate the shock pattern at a particular location. A steeper injection resulted in a similar shock pattern as observed with higher injection pressure and shallower injection.

- Depending on the injection location, counterrotating vortical structures can be generated in the core and the shear layer of the exhaust. The further the injection is placed inside the nozzle, the stronger are the vortex pairs penetrating the core. Contrary, the further the injection is move toward the nozzle exit, the stronger evolve the shear layer vortex pairs. The counterrotating vortical structures in the shear layer can strongly amplify the nozzle-near turbulence kinetic energy levels.

- The injected vortical structures decreased the turbulence kinetic energy development in the shear layer towards the end of the potential core.

- The injection pressure dominates the extent of the vortical structures, while the injection angle has a secondary effect.

- It has been shown that only at far over-expanded nozzle operating conditions, fluidic injection with large mass-flow rates can beneficially influence the thrust production.

\section{Acknowledgement}

The author would like to thank Dr. Gutmark and Dr. Cuppoletti at University of Cincinnati for sharing the experimental data.

\section{References}

${ }^{1}$ Rask, O., Kastner, J., and Gutmark, E., "Understanding how chevrons modify noise in supersonic jet with flight effects," AIAA journal, Vol. 49, No. 8, 2011, pp. 1569-1576.

${ }^{2}$ Saiyed, N. H., Mikkelsen, K. L., and Bridges, J. E., "Acoustics and thrust of quiet separate-flow highbypass-ratio nozzles," AIAA journal, Vol. 41, No. 3, 2003, pp. 372-378.

${ }^{3}$ Cuppoletti, D. R., Malla, B., Gutmark, E. J., Hafsteinsson, H. E., Eriksson, L.-E., and Prisell, E., "The Response of Supersonic Jet Noise Components to Fluidic Injection Parameters," 19th AIAA/CEAS Aeroacoustics Conference, 2196, 2013.

9 of 9 